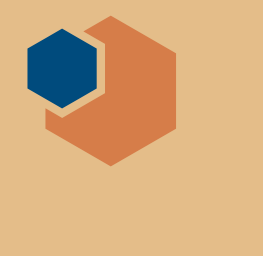

\title{
UK assesses impact of spending on academic research
}

$\mathrm{I}^{\mathrm{n}}$ direction that her administration will take, the United Kingdom's new Prime Minister, Theresa May, announced that by 2020 there will be an extra $£ 2$ billion per year of government investment for research and development (R\&D) "to ensure British business remains at the cutting edge of scientific and technological discovery." An increase of roughly a fifth, this is one of the largest boosts to R\&D spending in many years.

As the prime minister's speech suggests, governments fund research in the belief that there is a direct link between R\&D spending and economic growth. When trying to justify this expenditure at a time when the economy is in poor shape, the UK government can draw on the evidence of a recent exercise that $\mathrm{n}$ one of her first major speeches on the set out to determine the true impact of spending on academic research.

The Engineering and Physical Sciences Research Council (EPSRC), the UK's largest source of grants for research in engineering and the physical sciences, calculates that research it backed was behind more than 400 new businesses that employ 50,000 people and that have contributed some $£ 4$ billion to the economy. As Philip Nelson, Chief Executive of EPSRC puts it, "Research funded by the council can be associated with approximately [£60] billion of economic activity, including $£ 16$ billion of cost savings to the public and private sector."

Nelson's comments draw on EPSRC's analysis of the 1226 case studies directly relevant to its remit submitted to the Research Excellence Framework (REF) 2014. REF is a series of research benchmarking exercises, carried out every five or six years since 1986. For the first time, the Higher Education Funding Council for England (HEFCE), the body that ran REF2014, asked for case studies that described the impact of their research. Previous benchmarks sought details of the quality of the research outputs, judged by international comparisons, and information on the quality of the research "environment" that universities provided.

In materials research, EPSRC's analysis included, for example, case studies that described research at the University of Cambridge on the growth of gallium nitride that, the researchers said, helped the company Aixtron sell more than $£ 500$ million worth of equipment in the three years

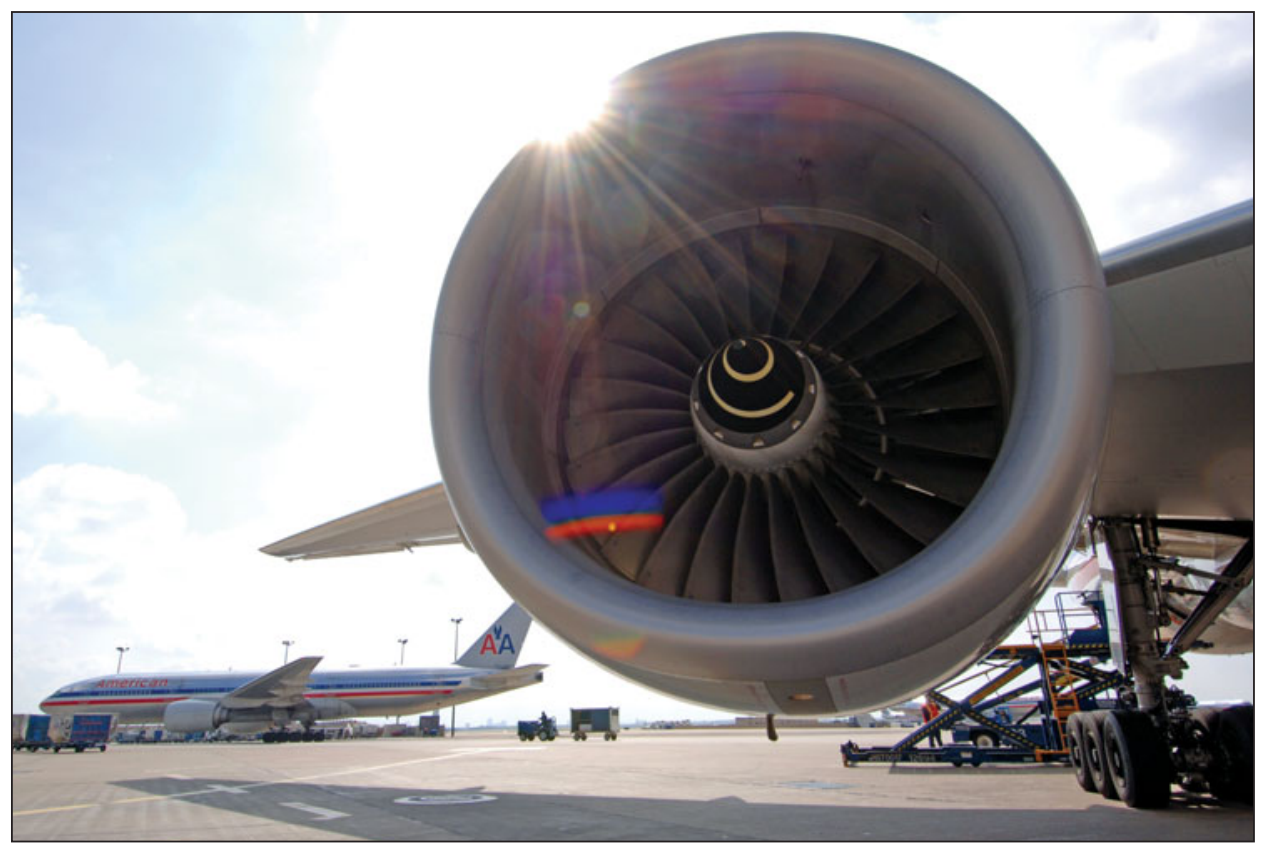

As a part of its submission to REF2014, Swansea University described research into the characterization of metallic alloys and ceramics that helped Rolls-Royce to define safe operational envelopes for titanium and nickel alloys utilized in the company's Trent 800 engines which power the Boeing 777. @ Rolls-Royce plc. running up to REF2014. The work also led to the manufacture of "the world's first commercially available LEDs [light-emitting diodes] on 6-inch [diameter] silicon."

Another case study for REF2014 reported on research at Imperial College London on engineering materials that delivered international standards and codes that have, the researchers say, saved industry "many millions of pounds" in applications in nuclear power stations, in composite materials in aircraft, plastic water pipes, and wind turbine blades. Again, the researchers talk of economic benefits in the millions. In line with REF2014's demand for real-world evidence of these 
benefits, Imperial College London said that EDF Energy, Airbus, RollsRoyce plc, and Vestas were just some of the companies that could corroborate these claims.

The original 1986 Research Selectivity Exercise set out to standardize the information available to government so it could be "more selective in its support for research ... to redistribute resources for research between institutions and to encourage redistribution within institutions towards work of special strength or promise." REF is more than just an exercise in assessing research quality: it determines how much universities receive from the $£ 1.6$ billion for "Quality Related" research that HEFCE hands out every year. This core funding for academic research sits alongside around $£ 3$ billion a year in targeted grants from research councils. EPSRC, the largest research center, distributes around $£ 900$ million a year.

In all, EPSRC calculates that it manages a research portfolio worth around $£ 4.6$ billion. In its analysis of the case studies in its domain, EPSRC says that "over $85 \%$ of the impact case studies in engineering and physical sciences involved research and/or researchers who were funded by EPSRC." These accounted for $£ 1$ billion of that portfolio, with a further $£ 1$ billion from other sources.

Not all impact is economic: the terms of reference for REF2014 talked of "any effect on, change or benefit to the economy, society, culture, public policy or services, health, the environment or quality of life, beyond academia." Money naturally looms large, partly because it is easier to estimate. It is also something that the academic community can use to demonstrate to politicians that university research delivers value for money.

As MRS Bulletin was going to press, HEFCE launched a public consultation on the next round of REF and sought the views of the research community on a series of questions. The funding council wants to receive responses to the consultation by the middle of March. It will then collect submissions for the next round of REF in 2020, and plans to seek input on the impact of research done between January 1, 2000 and the middle of 2020 .

The consultation document takes in the findings of an independent review of REF2014 led by Lord Nicholas Stern, whose earlier work had set the tone for the UK's debate on how to respond to climate change. The Stern review concluded that "[I]mpact as a principle is important and, even though it can and should be improved, it made a useful contribution as part of REF2014."

The next round of REF will also set out to tackle a long-standing issue for some researchers: the challenge of supporting, and assessing, interdisciplinary research. The consultation document says that REF2021 will set out to "encourage further the submission of interdisciplinary research." One key recommendation of the Stern review was that "[i]nstitutions should be given more flexibility to showcase their interdisciplinary and collaborative impacts by submitting 'institutional' level impact case studies, part of a new institutional level assessment." In an attempt to deal with this in REF2021, HEFCE also announced the creation of an Interdisciplinary Advisory Panel, chaired by Athene Donald D.B.E., professor of experimental physics at the University of Oxford. In her first comments on her role, Donald says that she hopes "to try to ensure that finally those working in new ways, in new combinations and with new insight and innovative approaches will be assessed fairly and equitably with those who sit neatly within the traditional silos."

Michael Kenward
BRICS Science, Technology and Innovation Partnershipbuilding responsive, inclusive, collective solutions

$\mathrm{R}$ esearch and innovation remain important instruments for enhancing competitiveness, accelerating growth, and promoting equitable growth within the BRICS nations (Brazil, Russia, India, China, and South Africa) and globally, says South Africa's Minister of Science and Technology Naledi Pandor.

Minister Pandor attended the 4th BRICS Science, Technology and Innovation Ministerial Meeting in Jaipur, India, last fall where the BRICS Ministers of Science and Technology lauded the outstanding progress in BRICS cooperation since their last gathering in 2015 .
This included the launch of a comprehensive framework program for funding research and innovation partnerships between the BRICS countries. The first call for proposals under this program attracted close to 400 proposals in areas such as advanced materials, nanotechnology, and the life sciences.

"I would like to emphasize the impact of the first pilot calls under the BRICS framework program for multilateral cooperation in research and innovation. It is, to quote an often used expression these days, a game changer in the field of international science and technology partnerships. What we have achieved in less than a year, other partnerships have taken decades to construct. The overwhelming response from our research and innovation communities to opportunities for cooperation provided under the program should encourage us to build on this success and to implement an ambitious second call without delay," Pandor says.

Pandor also emphasized the strategic importance of the proposed BRICS science and technology enterprise partnership. The BRICS partnership enterprise will focus on Science, Technology and Innovation on accelerating economic growth, providing an enabling framework to achieve this objective.

BRICS science and technology ministers will again meet in China in 2017. $\square$ 